\title{
Coste-efectividad del uso de esquemas de tratamiento farmacológico en pacientes con diagnóstico de diabetes mellitus tipo 2
}

\author{
Enrique Villarreal Ríos ${ }^{a}$, Karla Gabriela Romero Zamora, ${ }^{a, b}$, Emma Rosa Vargas Dazaa, \\ Liliana Galicia Rodrígueza , Lidia Martínez Gonzáleza y José Martín Ramos Lópeza
}

aUnidad de Investigación Epidemiológica y en Servicios de Salud Querétaro, Instituto Mexicano del Seguro Social, Querétaro, México.

b Unidad de Medicina Familiar $N^{\circ} 16$, Instituto Mexicano del Seguro Social, Querétaro, México.

Correspondencia: Enrique Villarreal Ríos, Zaragoza y 5 de Febrero, Colonia Centro, Querétaro, Querétaro de Arteaga, México. CP 76000. Correo electrónico: felibree@ infosel.net.mx.

Recibido el 19 de enero de 2012.

Aceptado para su publicación el 10 de Agosto de 2012.

\section{RESUMEN}

Objetivo. Determinar la evaluación coste-efectividad de los esquemas de manejo farmacológico en el paciente diabético.

Diseño. Diseño de coste-efectividad.

Emplazamiento: Unidades de Medicina Familiar 9, 13 y 16 del Instituto Mexicano del Seguro Social de la ciudad de Querétaro.

Participantes. Pacientes con diagnóstico de diabetes mellitus tipo 2, con tiempo de diagnóstico entre uno y cinco años.

Mediciones Principales. Se integraron tres grupos de 30 pacientes cada uno: Grupo 1: Tratamiento farmacológico con insulina NPH más metformina; Grupo 2: Tratamiento farmacológico con glibenclamida más metformina; Grupo 3: Tratamiento farmacológico con metformina. La técnica muestral fue por cuota, se utilizó como marco muestral los listados de pacientes con diabetes en las unidades de medicina familiar. Las variables estudiadas incluyeron características sociodemográficas, de salud, coste del uso de los servicios (medicina familiar, laboratorio y farmacia); y efectividad (glucemia promedio menor a $130 \mathrm{mg} / \mathrm{dl}$ ). Para el análisis estadístico se utilizó porcentajes, promedios y escenarios para coste-efectividad.

Resultados. En todos los escenarios la efectividad más alta corresponde al esquema de metformina, la efectividad fluctúa entre $48,3 \%$ y $100 \%$. El promedio anual de consultas en medicina familiar es más alto en el esquema de glibenclamida más metformina $(10,0)$. El coste más alto corresponde al esquema glibenclamida más metformina (173,67 euros). En el escenario de $130 \mathrm{mg} / \mathrm{dl}$ la mejor relación coste-efectividad se presenta en el esquema de metformina, lograr el $100 \%$ de efectividad tiene un coste de 1.657 euros en el esquema insulina más metformina, 695 euros en el esquema glibenclamida más metformina y de 334 euros en el esquema de metformina.

Conclusión. La mejor relación coste-efectividad corresponde al esquema de metformina.

Palabras clave. Diabetes mellitus tipo 2, Metformina, Glibenclamida, Insulinas, Tratamiento, Coste-efectividad.

\section{ABSTRACT}

Cost effectiveness of the use of pharmacological treatment regimens in patients diagnosed with type 1 Diabetes Mellitus

Objective. To determine the evaluation cost effectiveness of the schemes of pharmacologic therapy in the diabetic patient.

Design. Cost-effectiveness

Location. Familiar Medicine Units 9, 13 and 16 of the Mexican Institute of the Social Insurance of the city of Querétaro.

Participants. Patients with diagnosis of diabetes mellitus type 2, with time of diagnosis between one and five years.

Main measurements. Three groups of 30 patients integrated themselves each one: Group 1: Pharmacologic treatment with metformina and insulin NPH; Group 2: Pharmacologic treatment with glibenclamida and metformina; Group 3: Pharmacologic treatment with metformina. The technical sample was by quota, was used as frame sample the listings of patients with diabetes in the familiar medicine units. The studied variables included to characteristics sociodemographics, of health, cost of the use of the services (medicine familiar, to laboratory and pharmacia); and effectiveness (glycaemia smaller average to $130 \mathrm{mg} / \mathrm{dl}$ ). For statistical analysis was used percentage, averages and scenes for cost-effectiveness.

Results. In all the scenes the highest effectiveness corresponds to the metformina scheme, the effectiveness fluctuates between $48.3 \%$ and $100.0 \%$. The annual average of consultations in familiar medicine is highest in the scheme of glibenclamida and metformina (10.0). The highest cost corresponds to the scheme glibenclamida and metformina (€173.6). In the scene of 130 $\mathrm{mg} / \mathrm{dl}$ the best relation cost effectiveness appears in the metformina scheme, to obtain the $100 \%$ of effectiveness has a cost of $€ 1,657$ in scheme insulin and metformina, euros695 in the scheme glibenclamida and metformina, and e334 in scheme metformina.

Conclusion. Best relation cost effectiveness corresponds to the metformina scheme.

Key words. Diabetes mellitus type 2, Metformina, Glibenclamida, Insulin, Treatment, Costeffectiveness. 


\section{INTRODUCCIÓN}

En la actualidad, la diabetes mellitus tipo 2 (DM2) se considera un problema de salud pública, ante el aumento exponencial del número de casos, estimando que para el año 2030 existirán 366 millones de diabéticos ${ }^{1}$.

Para el sistema de salud, el objetivo del tratamiento de estos pacientes es el control metabólico que permita en el corto y largo plazo evitar las complicaciones y mantener la calidad de vida, basado en tres aspectos fundamentales: 1) el tratamiento farmacológico, 2) la actividad física y 3) la alimentación saludable? .

En particular, sobre el tratamiento farmacológico se ha identificado que en la práctica médica el manejo inicial está basado en la monoterapia con hipoglucemiantes orales, no obstante, el control metabólico raras veces se logra. Existen informes que señalan que aproximadamente entre el 30\% y el $48 \%$ de los diabéticos tiene la glucemia en cifras normales, y cuando la evaluación se realiza con hemoglobina glicosilada, el porcentaje de control se identifica entre $18 \%$ y el $50 \%{ }^{3}$. Este escenario ha propiciado la combinación de hipoglucemiantes, pero sin alcanzar los resultados deseados; situación que aumenta la probabilidad de progresión de complicaciones crónicas ${ }^{4,5}$.

No obstante lo anterior, lo que la evidencia científica ha demostrado es que el tratamiento basado en la insulina es la mejor alternativa para alcanzar el control metabólico. Al respecto existen informes que así lo señalan ${ }^{6}$.

Ante el panorama de la baja efectividad de los esquemas de tratamiento farmacológico, el sistema de salud, inmerso en la dinámica de la economía de la salud que pregona los recursos limitados, tiene la obligación de encontrar las alternativas que ofrezcan los mejores resultados al coste más bajo. En este contexto, los diseños de evaluación económica son la alternativa viable, en particular la evaluación coste-efectividad que permite analizar la relación de los costes con la obtención de resultados (efectividad) entre dos o más alternativas para elegir la mejor ${ }^{7,8}$.

En consecuencia, aunque la evidencia científica demuestre que la mejor alternativa de manejo en el paciente diabético es la insulina, en la práctica clínica se presenta otro panorama, por lo que el objetivo de este artículo es determinar la evaluación coste-efectividad de los esquemas de manejo farmacológico en el paciente diabético.

\section{MATERIAL Y MÉTODOS}

Se realizó un diseño de coste-efectividad en pacientes con diagnóstico de DM2, con tiempo de diagnóstico entre uno y cinco años, tratados por un médico familiar en las Unidades de Medicina Familiar 9, 13 y 16 del Instituto Mexicano del Seguro Social de la ciudad de Querétaro, de julio de 2011 a marzo de 2012.

Se formaron tres grupos de estudio de acuerdo a los esquemas de tratamiento farmacológico más utilizados en la consulta de medicina familiar:

- Grupo 1: Tratamiento farmacológico con insulina NPH más metformina.

- Grupo 2: Tratamiento farmacológico con glibenclamida más metformina.

- Grupo 3: Tratamiento farmacológico con metformina.

Se incluyeron pacientes con hipertensión, obesidad y cardiopatía, ya que estas patologías se encuentran asociadas en la mayoría de los pacientes diabéticos; fueron excluidos pacientes con antecedentes de nefropatía o desnutrición (índice de masa corporal menor a 19), así

\begin{tabular}{|c|c|c|c|}
\hline \multirow{2}{*}{ Características } & \multicolumn{3}{|c|}{ Esquemas de tratamiento } \\
\hline & Insulina más metformina & Glibenclamida más metformina & Metformina \\
\hline Edad (años) & $44,7(38,1-51,2)$ & $\begin{array}{c}\text { Promedios } \\
51,0(46,5-55,4)\end{array}$ & $52,8(48,0-57,5)$ \\
\hline Tiempo de evolución (meses) & $36,1(30,4-41,8)$ & $30,6(23,9-37,3)$ & $25,4(20,1-30,7)$ \\
\hline $\begin{array}{l}\text { Sexo (femenino) } \\
\text { Vida en pareja } \\
\text { Nivel escolar (secundaria o } \\
\text { menos) } \\
\text { Remuneración Económica } \\
\text { Hipertensión arterial } \\
\text { Obesidad } \\
\text { Cardiopatía } \\
\text { Otras Patologías }\end{array}$ & $\begin{array}{c}80,0(65,7-94,3) \\
70,0(55,6-86,4) \\
63,3(46,1-80,5) \\
53,3(35,4-71,2) \\
53,3(35,4-71,2) \\
30,0(13,6-46,4) \\
6,7(0,0-15,6) \\
6,7(0,0-15,6)\end{array}$ & $\begin{array}{c}\text { Porcentaje } \\
53,3(35,4-71,2) \\
92,9(83,7-100,0) \\
80,8(66,7-94,9) \\
67,9(51,2-84,6) \\
70,0(53,6-86,4) \\
40,0(22,5-57,5) \\
6,7(0,0-15,6) \\
6,7(0,0-15,6)\end{array}$ & $\begin{array}{r}60,0(42,5-77,5) \\
82,8(69,3-96,3) \\
72,0(55,9-88,1) \\
53,6(35,8-71,4) \\
66,7(49,8-83,6) \\
43,3(25,6-61,0) \\
6,7(0,0-15,6) \\
23,3(8,2-38,4)\end{array}$ \\
\hline
\end{tabular}

*Los valores entre paréntesis corresponden a intervalo de confianza del 95\%

Tabla 1. Características sociodemográficas y de salud por esquemas de tratamiento. 


\begin{tabular}{|c|c|c|c|}
\hline \multirow{3}{*}{ Escenario* $^{*}$} & \multicolumn{3}{|c|}{ Esquemas de tratamiento } \\
\hline & $\begin{array}{l}\text { Insulina más } \\
\text { metformina }\end{array}$ & $\begin{array}{c}\text { Glibenclamida más } \\
\text { metformina }\end{array}$ & Metformina \\
\hline & \multicolumn{3}{|c|}{ Efectividad (\%) } \\
\hline $130 \mathrm{mg} / \mathrm{dl}$ y menos & $10,0(0,0-20,7)$ & $25,0(9,5-40,5)$ & $48,3(30,4-66,2)$ \\
\hline $140 \mathrm{mg} / \mathrm{dl}$ y menos & $10,0(0,0-20,7)$ & $46,4(28,6-64,2)$ & $65,5(48,5-82,5)$ \\
\hline $150 \mathrm{mg} / \mathrm{dl}$ y menos & $20,0(5,7-34,3)$ & $53,6(35,8-71,4)$ & $69,0(52,4-85,6)$ \\
\hline $160 \mathrm{mg} / \mathrm{dl}$ y menos & $30,0(13,6-46,4)$ & $60,7(43,2-78,2)$ & $82,8(69,3-96,3)$ \\
\hline $170 \mathrm{mg} / \mathrm{dl}$ y menos & $30,0(13,6-46,4)$ & $75,0(59,5-90,5)$ & $89,7(78,8-100,0)$ \\
\hline $180 \mathrm{mg} / \mathrm{dl}$ y menos & $30,0(13,6-46,4)$ & $75,0(59,5-90,5)$ & $89,7(78,8-100,0)$ \\
\hline 190 mg/dl y menos & $46,7(28,8-64,6)$ & $78,6(63,9-93,3)$ & $89,7(78,8-100,0)$ \\
\hline 200 mg/dl y menos & $50,0(32,1-67,9)$ & $82,1(68,4-95,8)$ & $93,1(84,0-100,0)$ \\
\hline 240 mg/dl y menos & $76,7(61,6-91,8)$ & $89,3(78,2-100,0)$ & $100,0(100,0-100,0)$ \\
\hline
\end{tabular}

*Los escenarios los determina el nivel de glucosa en sangre

** Los valores entre paréntesis corresponden a intervalo de confianza del 95\%

Tabla 2. Efectividad de los esquemas de tratamiento por escenario.

\begin{tabular}{|c|c|c|c|c|c|c|c|}
\hline \multirow{3}{*}{ Servicios } & \multirow{3}{*}{ Coste $^{\star}$ unitario } & \multicolumn{6}{|c|}{ Esquemas de tratamiento } \\
\hline & & \multicolumn{3}{|c|}{ Uso de servicios } & \multicolumn{3}{|c|}{ Coste* $^{*}$} \\
\hline & & $\begin{array}{c}\text { Insulina más } \\
\text { metformina }\end{array}$ & $\begin{array}{c}\text { Glibenclamida más } \\
\text { metformina }\end{array}$ & Metformina & Insulina más metformina & $\begin{array}{l}\text { Glibenclamida más } \\
\text { metformina }\end{array}$ & Metformina \\
\hline & \multicolumn{7}{|c|}{ Medicina Familiar } \\
\hline Consultas & 11,38 & 8,84 & 10,08 & 9,53 & 100,64 & 114,76 & 108,49 \\
\hline Infección vías urinarias & 0,55 & 0,42 & 0,08 & 0,41 & 0,23 & 0,04 & 0,23 \\
\hline & \multicolumn{7}{|c|}{ Laboratorio } \\
\hline EGO & & 1,74 & 2,33 & 2,12 & 6,03 & 8,07 & 7,34 \\
\hline Glucosa & 4,47 & 3,00 & 3,33 & 2,94 & 13,40 & 14,87 & 13,13 \\
\hline Urea & 4,47 & 1,63 & 1,58 & 1,41 & 7,28 & 7,06 & 6,30 \\
\hline Creatinina & 4,47 & 1,89 & 1,83 & 1,88 & 8,44 & 8,17 & 8,40 \\
\hline Hemoglobina glicosilada & 7,45 & 1,32 & 2,00 & 1,47 & 9,83 & 14,90 & 10,95 \\
\hline Urocultivo & 13,17 & 0,00 & 0,00 & 0,06 & 0,00 & 0,00 & 0,79 \\
\hline & \multicolumn{7}{|c|}{ Farmacia } \\
\hline Insulina & 1,70 & 9,53 & 0,00 & 0,00 & 16,24 & 0,00 & 0,00 \\
\hline Glibenclamida & 0,10 & 0,00 & 6,25 & 0,00 & 0,00 & 0,63 & 0,00 \\
\hline Metformina & 0,25 & 14,74 & 20,92 & 23,88 & 3,64 & 5,17 & 5,90 \\
\hline Coste $^{*}$ & & & & & 165,73 & 173,67 & 161,53 \\
\hline
\end{tabular}

* Los costes se expresan en euros.

Tabla 3. Coste unitario, uso de los servicios y coste por esquema de tratamiento.

como pacientes en tratamiento con acarbosa y tratamientos esporádicos (un mes), en este último caso por no poder realizar una evaluación real de la efectividad del tratamiento.

El tamaño de la muestra ( $n=30$ por grupo) se calculó con la fórmula de comparación de dos proporciones con nivel de confianza del 95\% $\left(Z_{a}=1,64\right)$, poder de la prueba de $80 \%\left(Z_{a}=\right.$ $0,84)$, prevalencia de control glucémico con el tratamiento de insulina más metformina de $75 \%$ $\left(P_{1}=0,75\right)$ y prevalencia de control glucémico con el tratamiento de glibenclamida más metformina de $45 \%\left(P_{0}=0,45\right)$. La técnica muestral fue por cuota, se utilizaron como marco muestral los listados de pacientes con diabetes en las unidades de medicina familiar, buscando intencionadamente los pacientes con DM2.

Las variables estudiadas incluyeron:

- Características sociodemográficas (edad, sexo, escolaridad, ocupación y estado civil).

- Características de salud. Incluido tiempo de evolución de la diabetes mellitus y enfermedades asociadas.

- Coste del uso de los servicios. El coste se estimó para medicina familiar, laboratorio y medicamentos, relacionando el perfil de uso (número de ocasiones 


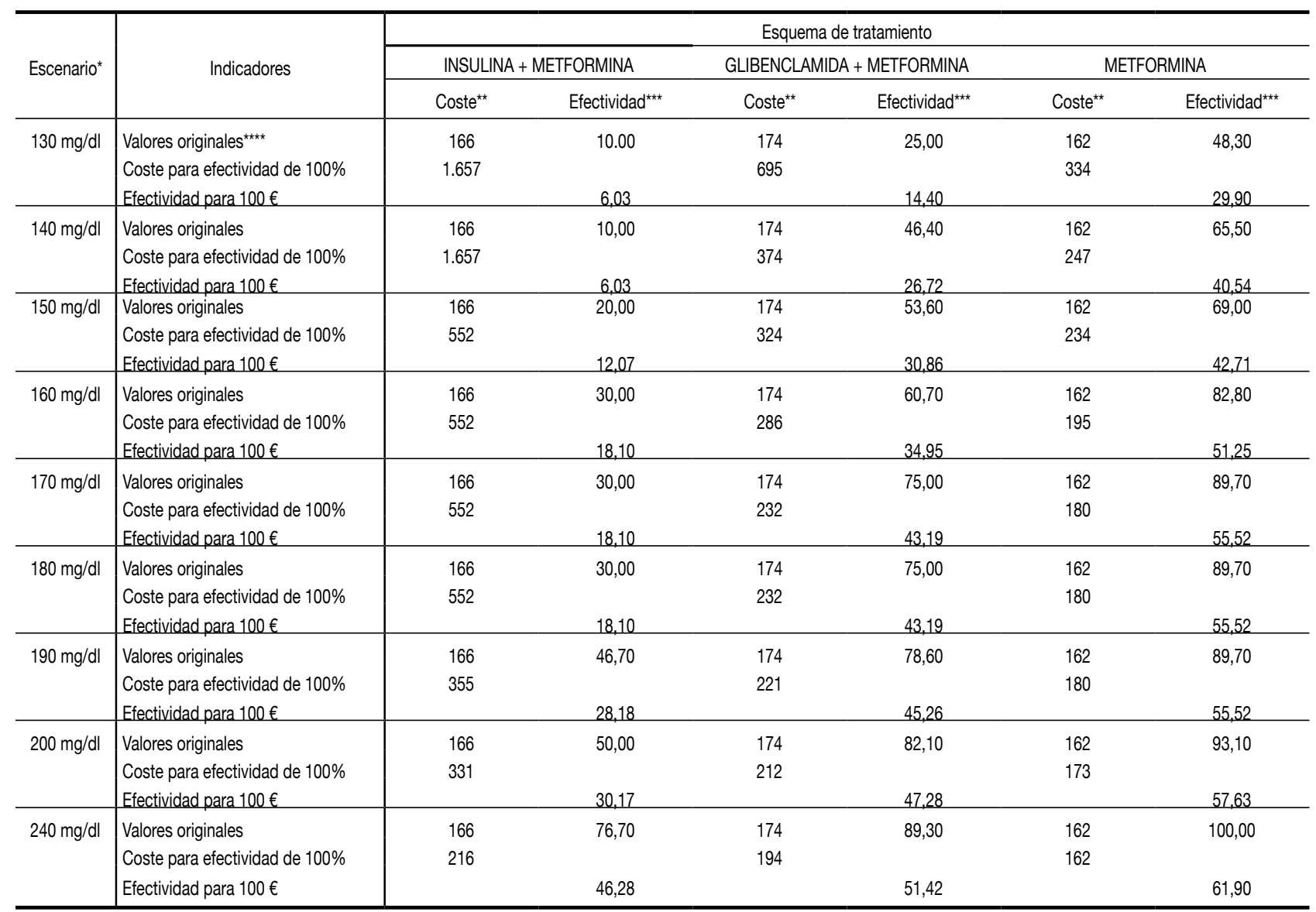

* El escenario está determinado por el nivel de glucosa en sangre. ${ }^{* *}$ El coste se expresa en euros. ${ }^{* * *}$ La efectividad se expresa en porcentaje. ${ }^{\star \star \star \star}$ Los valores originales corresponden al resultado de la medición original para cada escenario.

Tabla 4. Coste efectividad por esquemas de tratamiento para cada escenario.

que utilizó el servicio en el lapso de un año) con el coste unitario (coste de una atención).

- Perfil de uso. Para el perfil de uso se identificó en el expediente clínico el número de atenciones recibidas en el lapso de un año.

- Medicina familiar. Para medicina familiar se identificó el total de consultas otorgadas por control de la diabetes y el total de medicamentos prescritos para el tratamiento de infección de vías urinarias.

- Laboratorio. Se identificó el total de estudios de glucosa, examen general de orina, urea, creatinina, hemoglobina glicosilada y urocultivo.

- Medicamentos. Se contabilizó el total de medicamentos prescritos (insulina, glibenclamida y metformina).

- Coste unitario. El coste unitario se integró con el coste fijo (técnica de tiempos y movimientos) y el coste variable (microcosteo).
- Medicina familiar. Para medicina familiar el coste unitario se estimó mediante la técnica de tiempos y movimientos, se integró con la inversión en personal (operativo, directivo y administrativo), en servicios elementales (agua, electricidad, gas), equipo, mobiliario e infraestructura; se relacionó con el tiempo trabajado para conocer el coste por minuto de cada insumo. Paralelamente se midió el tiempo empleado en una atención promedio y la multiplicación del coste del minuto por el tiempo empleado en una atención se integró como coste unitario.

- Laboratorio. En laboratorio el coste unitario se estimó con la técnica de tiempos y movimientos, de la misma forma que para medicina familiar. A este valor se le sumó el coste de los reactivos necesarios para realizar cada uno de los estudios, para ello el consenso de expertos (microcosteo) definieron la cantidad de reactivos en cada prueba y se complementó con el coste de cada reactivo 


\begin{tabular}{cccc}
\hline & \multicolumn{2}{c}{ Ahorro $(€)$ por cada 100 pacientes tratados al sustituir el esquema de tratamiento } \\
\cline { 2 - 4 } Escenario & $\begin{array}{c}\text { Tratamiento con } \\
\text { "metformina" } \\
\text { en sustitución de } \\
\text { "insulina más metformina" }\end{array}$ & $\begin{array}{c}\text { Tratamiento con } \\
\text { "glibenclamida más } \\
\text { metformina" } \\
\text { en sustitución de }\end{array}$ & $\begin{array}{c}\text { Tratamiento con } \\
\text { "metformina" } \\
\text { en sustitución de } \\
\text { "insulina más metformina" }\end{array}$ \\
\hline $130 \mathrm{mg} / \mathrm{dl}$ & 1.322 .862 & 962.614 & 360.248 \\
$140 \mathrm{mg} / \mathrm{dl}$ & 1.410 .680 & 962.614 & 448.066 \\
$150 \mathrm{mg} / \mathrm{dl}$ & 594.546 & 504.635 & 89.911 \\
$160 \mathrm{mg} / \mathrm{dl}$ & 357.348 & 266.320 & 91.028 \\
$170 \mathrm{mg} / \mathrm{dl}$ & 372.354 & 320.871 & 51.483 \\
$180 \mathrm{mg} / \mathrm{dl}$ & 372.354 & 320.871 & 51.483 \\
$190 \mathrm{mg} / \mathrm{dl}$ & 174.805 & 133.927 & 40.877 \\
$200 \mathrm{mg} / \mathrm{dl}$ & 157.959 & 119.925 & 38.034 \\
$240 \mathrm{mg} / \mathrm{dl}$ & 54.547 & 21.597 & 32.950 \\
\hline
\end{tabular}

Tabla 5. Ahorro al sustituir el esquema de tratamiento en 1.000 pacientes con $100 \%$ de efectividad del tratamiento, para cada uno de los escenarios.

registrado en el departamento contable de la institución.

- Medicamentos. El coste de cada unidad de medicamento se identificó en el departamento contable de la institución.

- El coste del uso se integró con la multiplicación del perfil de uso por el coste unitario.

- Efectividad. La efectividad se midió a partir de la glucosa central, para ello se identificó el total de valores obtenidos en un año y se obtuvo el promedio de glucosa, este valor permitió identificar a los pacientes controlados y descontrolados, considerando como controlado (efectividad) aquellos con valor promedio menor a $130 \mathrm{mg} /$ dl.1 Se crearon escenarios para coste efectividad acorde a los niveles promedio de glucosa.

Para la recolección de datos se solicitaron los listados actualizados de pacientes portadores de DM2 en las unidades de medicina familiar, en ellos se buscó intencionadamente a pacientes que tuvieran prescrito cualquiera de los tres esquemas de tratamiento para formar los diferentes grupos. Ya identificados los posibles candidatos, se comprobó que en el expediente médico estuviera registrado el mismo tratamiento en las consultas recibidas durante el año; ante cualquier modificación del tratamiento inicial se eliminó ese expediente y se realizó una nueva búsqueda. La búsqueda de pacientes con esquemas de insulina fue más prolongada por su poca prescripción y mantenimiento dentro de los esquemas de tratamiento en pacientes con pocos años de haber sido diagnosticados. Esta información se obtuvo del expediente clínico, para ello se contó con la autorización del Comité de Investigación y del Director de la Unidad Médica.

Para el análisis estadístico se utilizaron porcentajes, promedios, intervalos de confianza del 95\%, escenarios para coste-efectividad de cada uno de los grupos establecidos de acuerdo a su nivel glucémico, análisis incremental con proyección del coste a 1.000 pacientes con $100 \%$ de efectividad y estimación del ahorro con la sustitución de los esquemas de tratamiento.

\section{RESULTADOS}

El promedio de edad para el esquema de insulina más metformina es 44,7 años (IC95\% 38,1-51,2), para el esquema glibenclamida más metformina 51,0 años (IC95\%; 46,5-55,4) y para el esquema metformina 52,8 años (IC95\% 48,0-57,5). El género femenino predomina en los tres esquemas de tratamiento, en el esquema de insulina más metformina 80,0\% (IC95\% 65,7-94,3), en glibenclamida más metformina 53,3\% (IC95\% $35,4-71,2)$ y en metformina $60,0 \%$ (IC95\% 42,5$77,5)$. En la tabla 1 se presentan el resto de las variables sociodemográficas.

El tiempo de evolución de la diabetes fluctúa entre 25,4 (IC95\% 20,1-30,7) meses y 36,1 (IC95\% $30,4-41,8)$ meses, dependiendo del esquema de 
tratamiento; esta información se presenta en la tabla 1. La hipertensión arterial, 83,3\% (IC95\% $70,0-96,6$ ), y la obesidad, 66,7\% (IC95\% 49,8$83,6)$, predominan en el esquema de glibenclamida y metformina; la cardiopatía, 41,2\% (IC95\% 23,6$58,8)$, predomina en el esquema de metformina. En la tabla 1 se presenta la prevalencia de estas patologías por cada esquema de manejo.

En todos los escenarios analizados la efectividad más alta corresponde al esquema de metformina, las cifras de efectividad fluctúan entre $48,3 \%$ (IC95\% 30,4-66,2) y 100\%; la efectividad más baja corresponde al esquema de insulina más metformina, en este caso los valores se encuentran entre 10,0\% (IC95\% 0,0-20,7) y 76,7\% (IC95\% $61,1-91,8)$. En la tabla 2 se presenta la efectividad por esquema y por escenario.

El promedio anual más alto de consultas en medicina familiar se identifica en el esquema glibenclamida más metformina (10,0), y el promedio más bajo corresponde al esquema insulina más metformina $(8,8)$. El coste más alto corresponde al esquema glibenclamida más metformina (173,7 euros) y el más bajo al esquema metformina (161,5 euros). En la tabla 3 se presenta el coste unitario por tipo de servicio, el uso de servicios y el coste por esquema de tratamiento.

En el escenario de $130 \mathrm{mg} / \mathrm{dl}$ la mejor relación coste-efectividad se presenta en el esquema de metformina, por cada 100 euros invertidos, la efectividad lograda en el esquema insulina más metformina es $6,0 \%$, en glibenclamida más metformina es $14,4 \%$ y en metformina es $29,9 \%$. En este mismo escenario, lograr el $100 \%$ de efectividad tiene un coste de 1.657 euros en el esquema insulina más metformina, 695 euros en el esquema glibenclamida más metformina y de 334 euros en el esquema de metformina. En la tabla 4 se presenta el análisis coste-efectividad de las tres alternativas de tratamiento para cada uno de los escenarios planteados.

Asumiendo el $100 \%$ de efectividad en los tres esquemas de tratamiento y proyectando el coste a 1.000 pacientes, en el escenario de $130 \mathrm{mg} / \mathrm{dl}$ sustituir el esquema insulina más metformina por metformina genera un ahorro de 1.322.862 euros; cuando el esquema de insulina más metformina se sustituye por glibenclamida más metformina el ahora es 962.614 euros; y cuando el esquema de glibenclamida más metformina se substituye por metformina, el ahorro es 360.248 euros. En el cuadro 5 se presenta el ahorro por cada uno de los escenarios propuestos.

\section{DISCUSIÓN}

El manejo farmacológico temprano en el paciente diabético en primer nivel de atención ha evolucionado, en la actualidad está ampliamente demostrada la importancia del esquema basado en insulina, no obstante es una realidad que en la práctica médica la población rechaza el uso de este medicamento de manera directa, negando la prescripción, o de manera indirecta, aceptando la prescripción pero rechazando su adherencia. Esta última conducta pareciera ser la más preocupante, ya que implica asignar recursos a un acción específica en la cual, la ausencia de adherencia propicia el uso ineficiente de los recursos asignados, situación crítica para el sistema de salud inmerso en la dinámica de la economía de la salud la cual pregona la escasez de recursos; de ahí la importancia de este artículo, que realiza en un contexto de práctica médica cotidiana (escenario muy diferente a la evaluación in vitro o al escenario controlado) la evaluación coste efectividad de los esquemas de tratamiento en el paciente diabético en primer nivel de atención 9 .

Los tres grupos que se comparan en este trabajo parecieran ser homogéneos, es verdad que en relación al tiempo de evolución existe diferencia estadística significativa entre el grupo de insulinametformina (36 meses) y metformina (25 meses), $y$ para el sexo entre insulina-metformina $y$ glibenclamida-metformina, no obstante se podría asumir que esta variabilidad no influye en el resultado.

Este trabajo, como todos los estudios, tiene limitaciones y aciertos. Dentro de las limitaciones se deberá mencionar el muestreo por cuota, es verdad que en él la posibilidad de los sujetos de observación de ser incluidos en el estudio no es la misma, con lo cual se incrementa la probabilidad de sesgos que distorsionen los resultados, aspecto que es necesario tener presente al momento de la interpretación de los resultados.

También es conveniente mencionar que cuando se realiza el análisis de sensibilidad debe estar claro que el supuesto adoptado contempla una relación lineal ente las variables, y es una realidad que este comportamiento no necesariamente es el que se presenta en la vida real; es decir, no por incrementar el gasto se va a disminuir proporcionalmente el nivel de glucosa $y$, en consecuencia, no necesariamente el porcentaje de efectividad disminuirá proporcionalmente. Este pareciera ser una limitación importante del análisis 
coste efectividad aquí presentado y en general de cualquiera de las modalidades de la evaluación económica (coste efectividad, coste utilidad o coste beneficio).

La efectividad de la insulina está ampliamente demostrada in vitro, incluso en grupos de pacientes con apego estricto al medicamento la efectividad continúa siendo la misma. Pero la evidencia ha demostrado una falta de apego al esquema de insulina, ésta es la realidad de los servicios de salud y necesariamente impacta en los costes de la atención y en el control metabólico del paciente diabético ${ }^{10,11,12}$. En consecuencia, no se propone evaluar la efectividad de la insulina en un escenario controlado, donde el apego sea del $100 \%$, el escenario propuesto para realizar el análisis contempla aquel en el cual la falta de apego es el común denominador. Se deberá reconocer que el apego es difícil de evaluar, dado que el resultado depende de la respuesta proporcionada por el paciente, quien generalmente afirma seguir el esquema de manejo prescrito ${ }^{12,13}$. En este contexto, la conducta del individuo ante los tratamientos farmacológicos, en particular al uso de la insulina, más que la efectividad farmacológica del medicamento pareciera que lo que determina la efectividad del esquema en el manejo es la adherencia ${ }^{13,14}$.

El control metabólico del paciente diabético incluye los niveles de glucemia, colesterol y triglicéridos, no obstante para fines de este estudio se tomó como indicador de efectividad el control de la glucemia. Es verdad que se trata de una decisión que puede ser criticada, pero también es verdad que en la práctica clínica, el médico familiar en el primer nivel de atención lo que evalúa es el control de las cifras de glucemia. Unido a ello, plantear una gama de escenarios contribuye a un análisis más amplio, propuesta que no omite la postura de la Asociación Americana de Diabetes ${ }^{15}$.

Lo que los resultados demuestran es que, al contrario de lo señalado por la literatura, la mayor efectividad se presenta para metformina ${ }^{16}$, con efectividad muy superior a la obtenida por la insulina-metformina e incluso, a la combinación de la metformina más glibenclamida, ésta es una realidad a la que se enfrenta el médico de familia en la práctica diaria. Este escenario obliga a una discusión sobre las posibles razones para obtener el resultado, entre ellas se puede argüir lo ya comentado en párrafos previos en relación a la insulina y el rechazo que la población tiene en torno al uso en etapas tempranas de la diabetes mellitus.
El coste de los esquemas de tratamiento propuestos es muy semejante en los tres casos, lo cual obliga a pensar que en el análisis coste efectividad lo que establece la diferencia entre ellos es la efectividad, tal y como se comprobó en los resultados. En los resultados se aprecia que a menor efectividad, es decir cuando el escenario analizado tiene por referencia valores de glucosas más altos, el coste disminuye, esto es verdad, obtener mejores resultados cuesta más.

El coste efectividad de los tratamientos analizados establece que en el grupo de pacientes manejados con hipoglucemiantes orales, se obtiene mayor efectividad a menor coste, en relación a los manejados con el esquema de insulina e hipoglucemiantes orales, escenario totalmente opuesto a la evidencia científica, la cual avala que el uso de insulina más metformina como tratamiento inicial obtiene mejores resultados ${ }^{6}$, pero en ésta población, como en muchas otras sucede lo contrario. En este contexto lo que se aprecia es el aumento de los costes, por el incremento en el uso de los servicios de salud y por el aumento en las dosis de medicamentos prescritos.

Lo que se puede señalar es que en la práctica médica no siempre se obtienen los mismos resultados que los alcanzados en escenarios controlados, y pareciera entonces que no basta con realizar la prescripción de los esquemas de tratamiento establecidos, es necesaria la participación del paciente en la búsqueda de controlar o restituir la salud, lo cual implica por parte del sistema de salud la educación de la población y por parte de la población la integración al proceso salud enfermedad y a la relación médico paciente, integración sin la cual los avances científicos tendrán poco éxito.

En conclusión, en el manejo de la diabetes mellitus se puede decir que en la práctica médica y en el contexto de la vida cotidiana del paciente diabético, la mejor relación coste efectividad corresponde a metformina, pero no se deberá olvidar que en un escenario controlado, la mejor relación costeefectividad corresponde a la insulina.

\section{BIBLIOGRAFÍA}

1. Vázquez J, Panduro A. Diabetes mellitus tipo 2: un problema epidemiológico y de emergencia en México. Investigación en Salud. 2001;3:18-26.

2. American Diabetes Association. Standards of medcal care in diabetes (ADA). Diabetes Care. 2011;34(suplemento 1):s16-s27. 
3. Sánchez-Migallón P. Control metabólico en pacientes diabéticos tipo 2: grado de control y nivel de conocimientos (Estudio AZUER). Rev Clin Med Fam. 2011;4(1):32-41.

4. García $F$, Rodríguez $M$, Malo $F$, Vázquez $F$, Modroño $M$, Fluiters E, et al. Guías clínicas - diabetes mellitus tipo 2 . Atención Primaria en la Red. [consultado el 2 de agosto de 2011] Disponible en: http://www.fisterra.com/guias2/ diabetes mellitus.asp.

5. Instituto Mexicano de Seguro Social. Guía de práctica clínica diagnóstico y tratamiento de la diabetes mellitus tipo 2 en el primer nivel de atención, 2009.

6. López M. La insulina en el tratamiento de la diabetes. Hospital Universitario "Virgen de las Nieves". [consultado el 2 agosto 2011] Disponible en: http://www.elendocrino.com/ linked/Archivos\%20profesionales/Terapia insulinica.pdf

7. Rodríguez-Ledesma MA, Vidal C. Conceptos básicos de economía de la salud para el médico general. Rev Med Inst Mex Seguro Soc. 2007;45(5):523-532.

8. Arredondo A, Nájera P, Leyva R. Atención médica ambulatoria en México: el coste para los usuarios. Salud Pública de Mex. 1999;41(1):18-26.

9. Enzo J. Evaluación socioeconómica del tratamiento de la diabetes mellitus tipo 2 en Argentina. Un análisis de coste-efectividad. Tesis de Maestría en Finanzas Públicas Provinciales y Municipales. Facultad de Ciencias Económicas. Universidad Nacional de la Plata. 2011 [consultado el 5 de agosto de 2011] Disponible en: http:// www.depeco.econo.unlp.edu.ar/doctrab/doc83.pdf.

10. Edwards K, Irons B, Xu T. Coste-efectividad de la insulina de acción intermedia o larga contra Exenatide en pacientes con diabetes mellitus tipo 2 no óptimamente controlados con antidiabéticos orales. Pharmacy Practice. 2006;4(3):129133.

11. Pech SW, Baeza JE, Ravell MJ. Factores que inciden en el fracaso del tratamiento del paciente diabètico en Tekax, Yucatán, México. Rev Esp Med Quir. 2010;15(4):211-215.

12. Durán-Varela BR, Rivera-Chavira B, Franco-Gallegos E. Apego al tratamiento farmacológico en pacientes con diagnóstico de diabetes mellitus tipo 2. Salud Pública Mex. 2001;43(3): 233-236.

13. Gómez-Rocha SA. Diabetes y estilo de vida. Rev Med Inst Mex Seguro Soc. 2010;48(5):539-542.

14. Vargas-Daza ER, Galicia-Rodríguez L, Turnbull-Plaza BE. Necesidades sentidas de los adultos mayores derechohabientes de cuidadores de medicina familiar. Rev Enfermería. 2009:1;32-41. [consultado el 30 de agosto de 2011] Disponible en: http://enfermeros.org/rev.

15. Ariza E, Camacho N, Londoño E, Niño C, Sequeda C, Solano C, et al. Factores asociados a control metabólico en pacientes diabéticos tipo 2. Salud Uninorte. 2005;21:2840. [consultado el 25 de agosto de 2011] Disponible en: http://ciruelo.uninorte.edu.co/pdf/salud_uninorte/21/4 Factores\%20asociados\%20a\%20control\%20metabolico. pdf.

16. Control de la glucosa: tratamiento con insulina. Guía Global para la diabetes tipo 2. Disponible en: http://www.idf.org/ webdata/docs/GGTD\%20ES\%2010\%20Tratamiento\%20 con\%20insulina.pdf. 